\title{
A General and Nonempirical Approach to the Determination of the Absolute Configuration of 1-Aryl-1,2-diols
}

\author{
Stefano Superchi, Daniele Casarini, Carmela Summa and Carlo Rosini* \\ Dipartimento di Chimica, Università della Basilicata, via N. Sauro 85, 85100, Potenza, Italy.
}

\section{Supplementary Material}

Full characterization and experimental procedures for compounds $\mathbf{1 b}-\mathbf{0}$ and $\mathbf{2 a - 0}$. NOE data from ${ }^{1} \mathrm{H}$ NMR analysis for $\mathbf{2 f}$. Computational data for compounds $(R)-\mathbf{2} \mathbf{i},(4 S, 5 R)-\mathbf{2 n}$, and $(4 S, 5 R)-\mathbf{2 o}(12$ pages).

General Procedures. Melting points are uncorrected. Optical rotations were measured at r.t. on a Jasco DIP-370 polarimeter. ${ }^{1} \mathrm{H}$ and ${ }^{13} \mathrm{C}$ NMR spectra were recorded on a Bruker Aspect 300 or a Varian INOVA 600 spectrometer. Enantiomeric excesses of the diols were determined by HPLC on a Daicel Chiralcel OD or Chiralcel OJ column. $\mathrm{CHCl}_{3}$ was distilled from $\mathrm{P}_{2} \mathrm{O}_{5}$ and stored over activated $4 \AA$ molecular sieves. Analytical TLC were performed on $0.20 \mathrm{~mm}$ silica gel plates Macherey-Nagel ALUGRAM SIL G/UV 254 and column chromatography was carried out with silica gel Macherey-Nagel 60 (70-230 mesh). 4-Biphenylboronic acid and diol (R)-1a were commercially available (Aldrich) while diols 1b-o were obtained via asymmetric dihydroxylation ${ }^{1}$ of the corresponding alkenes followed by recrystallization. The olefins 1-vinylnaphthalene and 9vinylanthracene were prepared from the corresponding aldehydes following a literature procedure. ${ }^{2}$ Absorption and CD spectra of compounds 2a-o were recorded on a JASCO J600 spectropolarimeter at room temperature, in freshly distilled THF, using $0.1 \mathrm{~mm}$ cells and concentrations of about $1 \times 10^{-3} \mathrm{M}$. During the measurement, the instrument was thoroughly purged with nitrogen.

\footnotetext{
${ }^{1}$ Kolb H. C.; Van Nieuwenhze, M. S.; Sharpless, K. B. Chem. Rev. 1994, 94, 2483.

${ }^{2}$ Antanaroli, S.; Berrettoni, M.; Cifarelli, G.; Lupi, A.; Marini-Bettolo, R. M.; Romeo, S. Gazz. Chim. Ital. 1992, 122 , 55.
} 
(R)-(-)-1-(4-Methoxyphenyl)ethane-1,2-diol (1b): Yield 73\%; mp 94-95 ${ }^{\circ} \mathrm{C}$, lit. $^{3} \mathrm{mp} 93-95^{\circ} \mathrm{C}$; $[\alpha]_{\mathrm{D}}=-37.0(c=0.9, \mathrm{EtOH})$, lit. $^{3}[\alpha]_{\mathrm{D}}=-35.0(c=1.0, \mathrm{EtOH})$, for $98 \%$ ee; ${ }^{1} \mathrm{H} \mathrm{NMR}(300 \mathrm{MHz}$, $\left.\mathrm{CDCl}_{3}\right) \delta 2.10($ br s, $1 \mathrm{H}), 2.50($ br s, $1 \mathrm{H}), 3.64\left(\mathrm{dd}, J^{\prime}=11.2 \mathrm{~Hz}, J^{\prime \prime}=8.0 \mathrm{~Hz}, 1 \mathrm{H}\right), 3.72\left(\mathrm{dd}, J^{\prime}=\right.$ $\left.11.2 \mathrm{~Hz}, J^{\prime \prime}=3.8 \mathrm{~Hz}, 1 \mathrm{H}\right), 3.81(\mathrm{~s}, 3 \mathrm{H}), 4.77\left(\mathrm{dd}, J^{\prime}=3.8 \mathrm{~Hz}, J^{\prime \prime}=8.0 \mathrm{~Hz}, 1 \mathrm{H}\right), 6.90(\mathrm{~d}, J=8.7 \mathrm{~Hz}$, 2H), 7.29 (d, $J=8.7 \mathrm{~Hz}, 2 \mathrm{H}$ ). Anal. Calcd for $\mathrm{C}_{9} \mathrm{H}_{12} \mathrm{O}_{3}: \mathrm{C}, 64.27$; H, 7.19. Found: C, 64.33; H, 7.25. Daicel Chiralcel OB column; $\lambda 220 \mathrm{~nm} ; n$-hexane: $i$-PrOH 95:5; flow $=1.0 \mathrm{~mL} / \mathrm{min}$; ee >99\%; first enantiomer eluted $(R)$.

(R)-(-)-1-(4-Trifluoromethylphenyl)ethane-1,2-diol (1c): Yield 82\%; mp 94-95 ${ }^{\circ} \mathrm{C}$, lit. $^{4} \mathrm{mp} 93-$ $94^{\circ} \mathrm{C} ;[\alpha]_{\mathrm{D}}=-43.8\left(c=1.0, \mathrm{CHCl}_{3}\right)$, lit. $^{4}[\alpha]_{\mathrm{D}}^{27}=+41.3\left(c=0.828, \mathrm{CHCl}_{3}\right)$ for $(S)-\mathbf{1 c} ;{ }^{1} \mathrm{H} \mathrm{NMR}$ $\left(300 \mathrm{MHz}, \mathrm{CDCl}_{3}\right) \delta 2.20(\mathrm{~s}, 1 \mathrm{H}), 2.82(\mathrm{~s}, 1 \mathrm{H}), 3.65\left(\mathrm{dd}, J^{\prime}=11.2 \mathrm{~Hz}, J^{\prime \prime}=7.9 \mathrm{~Hz}, 1 \mathrm{H}\right), 3.80(\mathrm{dd}$, $\left.J^{\prime}=11.2 \mathrm{~Hz}, J^{\prime \prime}=3.2 \mathrm{~Hz}, 1 \mathrm{H}\right), 4.89\left(\mathrm{dd}, J^{\prime}=3.2 \mathrm{~Hz}, J^{\prime \prime}=7.9 \mathrm{~Hz}, 1 \mathrm{H}\right), 7.49\left(\mathrm{~d}, J^{\prime}=8.3 \mathrm{~Hz}, 2 \mathrm{H}\right)$, 7.62 (d, $J=8.3 \mathrm{~Hz}, 2 \mathrm{H}$ ). Anal. Calcd for $\mathrm{C}_{9} \mathrm{H}_{9} \mathrm{~F}_{3} \mathrm{O}_{2}$ : C, 52.43; H, 4.40. Found: C, 52.27; H, 4.51. Daicel Chiralcel OD column; $\lambda 254 \mathrm{~nm}$; $n$-hexane: $i$-PrOH 94:6; flow $=0.8 \mathrm{~mL} / \mathrm{min}$; ee $>99 \%$; first enantiomer eluted $(R)$.

(R)-(-)-1-(4-Biphenyl)ethane-1,2-diol (1d): Yield 78\%; mp 151-152 ${ }^{\circ} \mathrm{C}$, lit. ${ }^{5} \mathrm{mp} 150-152^{\circ} \mathrm{C}$; $[\alpha]_{\mathrm{D}}$ $=-39.1\left(c=1.0, \mathrm{CHCl}_{3}\right)$, lit. $^{5}[\alpha]_{\mathrm{D}}=-38.9\left(c=1.03, \mathrm{CHCl}_{3}\right) ;{ }^{1} \mathrm{H} \mathrm{NMR}\left(300 \mathrm{MHz}, \mathrm{CDCl}_{3}\right) \delta 2.10$ (br s, 1H), 2.60 (br s, 1H), $3.72\left(\mathrm{dd}, J^{\prime}=11.2 \mathrm{~Hz}, J^{\prime \prime}=8.0 \mathrm{~Hz}, 1 \mathrm{H}\right), 3.82\left(\mathrm{dd}, J^{\prime}=11.2 \mathrm{~Hz}, J^{\prime \prime}=3.6\right.$ $\mathrm{Hz}, 1 \mathrm{H}), 4.89\left(\mathrm{dd}, J^{\prime}=3.6 \mathrm{~Hz}, J^{\prime}=8.0 \mathrm{~Hz}, 1 \mathrm{H}\right), 7.3-7.45(\mathrm{~m}, 1 \mathrm{H}), 7.45-7.55(\mathrm{~m}, 4 \mathrm{H}), 7.55-7.65$ (m, 4H). Anal. Calcd for $\mathrm{C}_{14} \mathrm{H}_{14} \mathrm{O}_{2}$ : C, 78.48; H, 6.59. Found: C, 78.27; H, 6.51. Daicel Chiralcel OJ column; $\lambda 220 \mathrm{~nm}$; $n$-hexane: $i$-PrOH 90:10; flow $=0.8 \mathrm{~mL} / \mathrm{min}$; ee $>99 \%$; first enantiomer eluted $(R)$.

(R)-(-)-1-(3-Bromophenyl)ethane-1,2-diol (1e): Yield 72\%; $[\alpha]_{\mathrm{D}}=-33.5\left(c=1.12, \mathrm{CHCl}_{3}\right) ;{ }^{1} \mathrm{H}$ NMR $\left(300 \mathrm{MHz}, \mathrm{CDCl}_{3}\right) \delta 2.68(\mathrm{~s}, 1 \mathrm{H}), 3.20(\mathrm{~s}, 1 \mathrm{H}), 3.65\left(\mathrm{dd}, J^{\prime}=11.0 \mathrm{~Hz}, J^{\prime \prime}=7.9 \mathrm{~Hz}, 1 \mathrm{H}\right), 3.74$ $(\mathrm{d}, J=11.0 \mathrm{~Hz}, 1 \mathrm{H}), 4.77\left(\mathrm{dd}, J^{\prime}=2.9 \mathrm{~Hz}, J^{\prime}=7.9 \mathrm{~Hz}, 1 \mathrm{H}\right), 7.2-7.3(\mathrm{~m}, 2 \mathrm{H}), 7.4-7.5(\mathrm{~m}, 1 \mathrm{H}), 7.52$ $(\mathrm{s}, 1 \mathrm{H}) .{ }^{13} \mathrm{C} \mathrm{NMR}\left(\mathrm{CDCl}_{3}\right) \delta 67.7,73.9,122.6,124.7,129.2,130.0,131.0,142.8$. Anal. Calcd for $\mathrm{C}_{8} \mathrm{H}_{9} \mathrm{BrO}_{2}$ : C, 44.27; H, 4.18. Found: C, 44.35; H, 4.13. Daicel Chiralcel OD column; $\lambda 220 \mathrm{~nm} ; n$ hexane: $i-\mathrm{PrOH} 97: 3$; flow $=0.5 \mathrm{~mL} / \mathrm{min}$; ee $98 \%$; first enantiomer eluted $(R)$.

\footnotetext{
${ }^{3}$ Ferraboschi, P.; Grisenti, P.; Manzocchi, A.; Santaniello, E. J. Chem. Soc., Perkin Trans. 1 1990, 2469.

${ }^{4}$ Hirose, K.; Ogasahara, K.; Nishioka, K.; Tobe, Y.; Naemura, K.; J. Chem. Soc., Perkin Trans. 2 2000, 1984.

${ }^{5}$ Miao, G.; Rossiter, B. E. J. Org. Chem. 1995, 60, 8424.
} 
(R)-(-)-1-(2-Bromophenyl)ethane-1,2-diol (1f): Yield 80\%; mp 119-120 ${ }^{\circ} \mathrm{C}$, lit. ${ }^{6} \mathrm{mp} 118-120^{\circ} \mathrm{C}$; $[\alpha]_{\mathrm{D}}=-57.7\left(c=1.02, \mathrm{CHCl}_{3}\right) ;$ lit. $^{6}[\alpha]_{\mathrm{D}}=-7.5\left(c=0.99, \mathrm{CHCl}_{3}\right) ;{ }^{1} \mathrm{H} \mathrm{NMR}\left(300 \mathrm{MHz}, \mathrm{CDCl}_{3}\right) \delta$ $1.70(\mathrm{~s}, 1 \mathrm{H}), 2.25(\mathrm{~s}, 1 \mathrm{H}), 3.56\left(\mathrm{dd}, J^{\prime}=11.3 \mathrm{~Hz}, J^{\prime \prime}=8.0 \mathrm{~Hz}, 1 \mathrm{H}\right), 3.90\left(\mathrm{dd}, J^{\prime}=11.3 \mathrm{~Hz}, J^{\prime \prime}=2.8\right.$ $\mathrm{Hz}, 1 \mathrm{H}), 5.20\left(\mathrm{dd}, J^{\prime}=8.0 \mathrm{~Hz}, J^{\prime \prime}=2.8 \mathrm{~Hz}, 1 \mathrm{H}\right), 7.1-7.6(\mathrm{~m}, 4 \mathrm{H})$. Anal. Calcd for $\mathrm{C}_{8} \mathrm{H}_{9} \mathrm{BrO}_{2}: \mathrm{C}$, 44.27; H, 4.18. Found: C, 44.23; H, 4.22. Daicel Chiralcel OJ column; $\lambda 220 \mathrm{~nm}$; $n$-hexane: $i$-PrOH 95:5; flow $=0.5 \mathrm{~mL} / \mathrm{min}$; ee $96 \%$; first enantiomer eluted $(R)$.

(R)-(-)-1-(2-Naphthyl)ethane-1,2-diol (1g): Yield 85\%; mp 132-133 ${ }^{\circ} \mathrm{C}$, lit. ${ }^{7} \mathrm{mp} 126-127^{\circ} \mathrm{C}$; $[\alpha]_{\mathrm{D}}$ $=-32.0(c=1.01, \mathrm{EtOH})$, lit. $^{7}[\alpha]_{\mathrm{D}}=-33.7(c=1.2, \mathrm{EtOH}) ;{ }^{1} \mathrm{H} \mathrm{NMR}\left(300 \mathrm{MHz}, \mathrm{CDCl}_{3}\right) \delta 1.80$ (br s, 2H), $3.76\left(\mathrm{dd}, J^{\prime}=8.0 \mathrm{~Hz}, J^{\prime \prime}=11.2 \mathrm{~Hz}, 1 \mathrm{H}\right), 3.87\left(\mathrm{dd}, J^{\prime}=11.2 \mathrm{~Hz}, J^{\prime \prime}=3.5 \mathrm{~Hz}, 1 \mathrm{H}\right), 5.01$ $\left(\mathrm{dd}, J^{\prime}=3.5 \mathrm{~Hz}, J^{\prime \prime}=8.0 \mathrm{~Hz}, 1 \mathrm{H}\right), 7.4-7.6(\mathrm{~m}, 3 \mathrm{H}), 7.8-8.0(\mathrm{~m}, 4 \mathrm{H})$. Anal. Calcd for $\mathrm{C}_{12} \mathrm{H}_{12} \mathrm{O}_{2}: \mathrm{C}_{\text {, }}$ 76.57; H, 6.43. Found: C, 76.49; H, 6.61. Daicel Chiralcel OD column; $\lambda 254 \mathrm{~nm} ; n$-hexane: $i$-PrOH 90:10; flow $=1.0 \mathrm{~mL} / \mathrm{min}$; ee $>99 \%$; first enantiomer eluted $(R)$.

(R)-(-)-1-(1-Naphthyl)ethane-1,2-diol (1h): Yield 75\%; mp 84-85 ${ }^{\circ}$, lit. ${ }^{5} \mathrm{mp} 85-86^{\circ} \mathrm{C}$; $[\alpha]_{\mathrm{D}}=-$ $73.9(c=1.0, \mathrm{MeOH})$, lit. $^{5}[\alpha]_{\mathrm{D}}=-76.8(c=1.0, \mathrm{MeOH}) ;{ }^{1} \mathrm{H} \mathrm{NMR}\left(300 \mathrm{MHz}, \mathrm{CDCl}_{3}\right) \delta 2.00(\mathrm{br} \mathrm{s}$, $2 \mathrm{H}), 3.79\left(\mathrm{dd}, J^{\prime}=10.0 \mathrm{~Hz}, J^{\prime \prime}=8.8 \mathrm{~Hz}, 1 \mathrm{H}\right), 3.97\left(\mathrm{dd}, J^{\prime}=1.6 \mathrm{~Hz}, J^{\prime \prime}=10.0 \mathrm{~Hz}, 1 \mathrm{H}\right), 5.64(\mathrm{dd}$, $\left.J^{\prime}=8.8 \mathrm{~Hz}, J^{\prime \prime}=1.6 \mathrm{~Hz}, 1 \mathrm{H}\right), 7.4-7.6(\mathrm{~m}, 3 \mathrm{H}), 7.70(\mathrm{~d}, J=7.0 \mathrm{~Hz}, 1 \mathrm{H}), 7.80(\mathrm{~d}, J=9.2 \mathrm{~Hz}, 1 \mathrm{H})$, $7.88(\mathrm{~d}, J=8.2 \mathrm{~Hz}, 1 \mathrm{H}), 8.06(\mathrm{~d}, J=7.6 \mathrm{~Hz}, 1 \mathrm{H})$. Anal. Calcd for $\mathrm{C}_{12} \mathrm{H}_{12} \mathrm{O}_{2}: \mathrm{C}, 76.57 ; \mathrm{H}, 6.43$. Found: C, 76.55; H, 6.58. Daicel Chiralcel OB column; $\lambda 254 \mathrm{~nm}$; $n$-hexane: $i$-PrOH 90:10; flow $=$ $0.5 \mathrm{~mL} / \mathrm{min}$; ee $96 \%$; first enantiomer eluted $(R)$.

(R)-(-)-1-(9-Anthryl)ethane-1,2-diol (1i): Yield 72\%; mp 132-133 ${ }^{\circ} \mathrm{C}$, lit. ${ }^{8} \mathrm{mp} 134-135^{\circ} \mathrm{C}$; $[\alpha]_{\mathrm{D}}=$ $-7.5(c=1.05, \mathrm{EtOH})$, lit. $^{8}[\alpha]_{\mathrm{D}}=-6.9(c=0.22, \mathrm{EtOH}) ;{ }^{1} \mathrm{H}$ NMR $\left(300 \mathrm{MHZ}, \mathrm{CDCl}_{3}\right) \delta 2.34(\mathrm{br}$ s, $2 \mathrm{H}), 3.90\left(\mathrm{dd}, J^{\prime}=3.4 \mathrm{~Hz}, J^{\prime \prime}=10.1 \mathrm{~Hz}, 1 \mathrm{H}\right), 4.46\left(\mathrm{t}, J^{\prime}=10.1 \mathrm{~Hz}, 1 \mathrm{H}\right), 6.37\left(\mathrm{dd}, J^{\prime}=3.6 \mathrm{~Hz}, J^{\prime \prime}\right.$ $=11.3 \mathrm{~Hz}, 1 \mathrm{H}), 7.4-7.6(\mathrm{~m}, 4 \mathrm{H}), 8.0(\mathrm{~d}, J=7.5 \mathrm{~Hz}, 2 \mathrm{H}), 8.44(\mathrm{~s}, 1 \mathrm{H}), 8.66(\mathrm{~d}, J=8.1 \mathrm{~Hz}, 2 \mathrm{H})$; ${ }^{13} \mathrm{C}$ NMR $\left(75 \mathrm{MHz}, \mathrm{CDCl}_{3}\right) \delta 66.1,72.5,124.6,124.9,125.8,128.6,129.3,129.9,130.3,131.6$. Anal. Calcd for $\mathrm{C}_{16} \mathrm{H}_{14} \mathrm{O}_{2}$ : C, 80.65; H, 5.92. Found: C, 80.37; H, 6.12. Daicel Chiralcel OD column; $\lambda 254 \mathrm{~nm}$; $n$-hexane: $i$-PrOH 90:10; flow $=0.5 \mathrm{~mL} / \mathrm{min}$; ee $96 \%$; first enantiomer eluted $(R)$.

\footnotetext{
${ }^{6}$ Konigsberger, K.; Hudlicky, T. Tetrahedron: Asymmetry 1993, 4, 2469.

${ }^{7}$ Howe, R.; Moore, R. H.; Rao, B. S. J. Med. Chem. 1973, 16, 1020.

${ }^{8}$ Corey, E. J.; Noe, M. C. J. Am. Chem. Soc. 1996, $118,11038$.
} 
(2S,3R)-(-)-Methyl-2,3-dihydroxy-3-phenylpropionate (1j): Yield 75\%; mp 84-85 ${ }^{\circ} \mathrm{C}$, lit. ${ }^{9} \mathrm{mp}$ $85-85.5^{\circ} \mathrm{C} ;[\alpha]_{\mathrm{D}}=-10.5\left(c=1.0, \mathrm{CHCl}_{3}\right)$, lit. $^{9}[\alpha]_{\mathrm{D}}=-10.7\left(c=1.1, \mathrm{CHCl}_{3}\right) ;{ }^{1} \mathrm{H} \mathrm{NMR}(300 \mathrm{MHz}$, $\left.\mathrm{CDCl}_{3}\right) \delta 2.80($ br s, $1 \mathrm{H}), 3.15($ br s, $1 \mathrm{H}), 3.81(\mathrm{~s}, 3 \mathrm{H}), 4.38(\mathrm{~d}, J=2.6 \mathrm{~Hz}, 1 \mathrm{H}), 5.02(\mathrm{~d}, J=2.6 \mathrm{~Hz}$, 1H), 7.2-7.4 (m, 5H). Anal. Calcd for $\mathrm{C}_{10} \mathrm{H}_{12} \mathrm{O}_{4}$ : C, 61.21; H, 6.17. Found: C, 61.19; H, 6.15. Daicel Chiralcel OJ column, $\lambda=220 \mathrm{~nm}, n$-hexane : $i$-PrOH $=90: 10$; flow $0.5 \mathrm{~mL} / \mathrm{min}$; ee $>98 \%$, first enantiomer eluted $(2 R, 3 S)$.

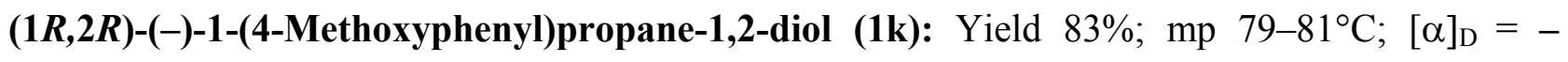
$32.08(c=1.01, \mathrm{MeOH})$, lit. $^{10}[\alpha]_{\mathrm{D}}=-34.67\left(c=1.3, \mathrm{CHCl}_{3}\right) ;{ }^{1} \mathrm{H} \mathrm{NMR}\left(300 \mathrm{MHz}, \mathrm{CDCl}_{3}\right) \delta 1.02$ $(\mathrm{d}, J=6.3 \mathrm{~Hz}, 3 \mathrm{H}), 2.69(\mathrm{~s}, 2 \mathrm{H}), 3.80(\mathrm{~s}, 3 \mathrm{H}), 3.81\left(\mathrm{dq}, J^{\prime}=6.3 \mathrm{~Hz}, J^{\prime}=7.7 \mathrm{~Hz}, 1 \mathrm{H}\right), 4.30(\mathrm{~d}, J=$ $7.7 \mathrm{~Hz}, 1 \mathrm{H}), 6.88(\mathrm{~d}, J=8.7 \mathrm{~Hz}, 2 \mathrm{H}), 7.25(\mathrm{~d}, J=8.7 \mathrm{~Hz}, 2 \mathrm{H})$. Anal. Calcd for $\mathrm{C}_{10} \mathrm{H}_{14} \mathrm{O}_{3}: \mathrm{C}, 65.92$; H, 7.74. Found: C, 65.85; H, 7.87. Daicel Chiralcel OJ column; $\lambda 254 \mathrm{~nm} ; n$-hexane: $i$-PrOH 90:10; flow $=0.5 \mathrm{~mL} / \mathrm{min}$; ee $>99 \%$; first enantiomer eluted $(1 R, 2 R)$.

(1R,2S)-(-)-1-(4-Methoxyphenyl)propane-1,2-diol (11): Yield 80\%; mp 114.1-116.6 ${ }^{\circ} \mathrm{C} ;[\alpha]_{\mathrm{D}}=-$ $4.0\left(c=1.05, \mathrm{CHCl}_{3}\right) ;{ }^{1} \mathrm{H} \mathrm{NMR}\left(300 \mathrm{MHz}, \mathrm{CDCl}_{3}\right) \delta 1.05(\mathrm{~d}, J=6.5 \mathrm{~Hz}, 3 \mathrm{H}), 1.8($ br s, $1 \mathrm{H}), 2.4$ (br s, 1H), $3.81(\mathrm{~s}, 3 \mathrm{H}), 3.98\left(\mathrm{dq}, J^{\prime}=6.5 \mathrm{~Hz}, J^{\prime \prime}=4.5 \mathrm{~Hz}, 1 \mathrm{H}\right), 4.60(\mathrm{~d}, J=4.5 \mathrm{~Hz}, 1 \mathrm{H}), 6.90(\mathrm{~d}, J$ $=8.7 \mathrm{~Hz}, 2 \mathrm{H}), 7.28(\mathrm{~d}, J=8.7 \mathrm{~Hz}, 2 \mathrm{H})$. Anal. Calcd for $\mathrm{C}_{10} \mathrm{H}_{14} \mathrm{O}_{3}: \mathrm{C}, 65.92 ; \mathrm{H}, 7.74$. Found: $\mathrm{C}$, 65.88; H, 7.77. Daicel Chiralcel OD column; $\lambda 254 \mathrm{~nm}$; $n$-hexane: $i$-PrOH 95:5; flow $=0.5 \mathrm{~mL} / \mathrm{min}$; ee $40 \%$; first enantiomer eluted $(1 R, 2 S)$.

(1S,2R)-(-)-1,2-Dihydroxyindene (1n): Yield 70\%; mp 98-100 $\mathrm{C}$, lit. ${ }^{11} \mathrm{mp} 99-100^{\circ} \mathrm{C} ;[\alpha]_{\mathrm{D}}=-$ $27.8\left(c=1.0, \mathrm{CHCl}_{3}\right)$, lit. $^{11}[\alpha]^{25}{ }_{\mathrm{D}}=-51.0\left(c=0.4, \mathrm{CHCl}_{3}\right) ;{ }^{1} \mathrm{H} \mathrm{NMR}\left(300 \mathrm{MHz}, \mathrm{CDCl}_{3}\right) \delta 2.50(\mathrm{~s}$, 2H), $2.96\left(\mathrm{dd}, J^{\prime}=16.3 \mathrm{~Hz}, J^{\prime \prime}=3.5 \mathrm{~Hz}, 1 \mathrm{H}\right), 3.12\left(\mathrm{dd}, J^{\prime}=16.3 \mathrm{~Hz}, J^{\prime \prime}=5.7 \mathrm{~Hz}, 1 \mathrm{H}\right), 4.50(\mathrm{~m}$, 1H), $5.00(\mathrm{~d}, J=4.9 \mathrm{~Hz}, 1 \mathrm{H}), 7.2-7.3(\mathrm{~m}, 3 \mathrm{H}), 7.4-7.5(\mathrm{~m}, 1 \mathrm{H})$. Daicel Chiralcel OJ column, $\lambda 220$ nm, $n$-hexane: $i$-PrOH 95:5; flow $0.8 \mathrm{~mL} / \mathrm{min}$; ee $57 \%$; first enantiomer eluted $(1 R, 2 S)$.

(1S,2R)-(+)-1,2,3,4-Tetrahydro-1,2-dihydroxynaphthalene (10): Yield 65\%; $[\alpha]_{\mathrm{D}}=+9.7(c=$ 1.07, EtOH), lit. ${ }^{12}[\alpha]_{\mathrm{D}}=-18.6(c=0.694, \mathrm{EtOH})$ for an $(1 R, 2 S)$ sample, ee $40 \% ;{ }^{1} \mathrm{H}$ NMR $(300$ $\left.\mathrm{MHz} \mathrm{CDCl}_{3}\right) \delta 1.8-2.1(\mathrm{~m}, 2 \mathrm{H}), 2.6-2.8(\mathrm{~m}, 1 \mathrm{H}), 2.8-3.1(\mathrm{~m}, 1 \mathrm{H}), 3.1(\mathrm{br} \mathrm{s}, 2 \mathrm{H}), 3.8-4.0(\mathrm{~m}, 1 \mathrm{H})$, $4.59(\mathrm{~d}, J=3.4 \mathrm{~Hz}, 1 \mathrm{H}), 6.9-7.5(\mathrm{~m}, 4 \mathrm{H}) ;{ }^{13} \mathrm{C} \mathrm{NMR}\left(75 \mathrm{MHz}, \mathrm{CDCl}_{3}\right) \delta 26.2,26.9,69.5,69.9$,

\footnotetext{
${ }^{9}$ Denis, J. -N.; Correa, A.; Greene, A. E. J. Org. Chem. 1990, 55, 1957.

${ }^{10}$ Ishida, T.; Bounds, S. V. J.; Caldwell, J.; Drake, A. F.; Takeshita, M. Tetrahedron: Asymmetry 1996, 7, 3113.

${ }^{11}$ Imuta, M.; Ziffer, H. J. Org. Chem. 1978, 43, 4540.

${ }^{12}$ Becker, H.; King, S. B.; Taniguchi, M.; Vanhessche, K. P. M. Sharpless, K. B. J. Org. Chem. 1995, 60, 3940.
} 
126.4, 128.1, 128.5, 129.6, 136.2, 136.4. Daicel Chiralcel OJ column, $\lambda 220 \mathrm{~nm}, n$-hexane: $i$-PrOH 95:5; flow $0.8 \mathrm{~mL} / \mathrm{min}$; ee $50 \%$; first enantiomer eluted $(1 S, 2 R)$.

\section{General Procedure for the Synthesis of Boronic Esters.}

To a solution of the diol $(0.36 \mathrm{mmol})$ in $\mathrm{CHCl}_{3}(5 \mathrm{~mL})$ were added 4-biphenylboronic acid $(0.44$ mmol), and activated $4 \AA$ molecular sieves. The mixture was left stirring at r.t. overnight, then filtered and the solvent evaporated. The recovered residue was purified by column chromatography $\left(\mathrm{CHCl}_{3}\right)$ affording the pure boronate.

(R)-(-)-2-(4-Biphenyl)-4-phenyl-1,3,2-dioxaborolane (2a): Yield 89\%; glassy solid; $[\alpha]_{\mathrm{D}}=-$ $102.1(c=1.07, \mathrm{THF}) ;{ }^{1} \mathrm{H}$ NMR $\left(300 \mathrm{MHz}, \mathrm{CDCl}_{3}\right) \delta 4.22\left(\mathrm{dd}, J^{\prime}=7.7 \mathrm{~Hz}, J^{\prime \prime}=8.9 \mathrm{~Hz}, 1 \mathrm{H}\right), 4.77$ $\left(\mathrm{dd}, J^{\prime}=8.0 \mathrm{~Hz}, J^{\prime \prime}=8.9 \mathrm{~Hz}, 1 \mathrm{H}\right), 5.62\left(\mathrm{dd}, J^{\prime}=7.7 \mathrm{~Hz}, J^{\prime \prime}=8.0 \mathrm{~Hz}, 1 \mathrm{H}\right), 7.3-7.5(\mathrm{~m}, 8 \mathrm{H}), 7.5-7.7$ (m, 4H), 7.97 (d, $J=8.0 \mathrm{~Hz}, 2 \mathrm{H})$. Anal. Calcd for $\mathrm{C}_{20} \mathrm{H}_{17} \mathrm{O}_{2} \mathrm{~B}: \mathrm{C}, 80.03$; H, 5.71. Found: C, 80.19; H, 5.63 .

(R)-(-)-2-(4-Biphenyl)-4-(4'-Methoxyphenyl)-1,3,2-dioxaborolane (2b): Yield 60\%; glassy solid; $[\alpha]_{\mathrm{D}}=-17.6(c=1.095, \mathrm{THF}) ;{ }^{1} \mathrm{H} \mathrm{NMR}\left(300 \mathrm{MHz}, \mathrm{CDCl}_{3}\right) \delta 3.81(\mathrm{~s}, 3 \mathrm{H}), 4.20\left(\mathrm{dd}, J^{\prime}=7.8 \mathrm{~Hz}\right.$, $\left.J^{\prime \prime}=8.8 \mathrm{~Hz}, 1 \mathrm{H}\right), 4.70\left(\mathrm{dd}, J^{\prime}=J^{\prime \prime}=8.6 \mathrm{~Hz}, 1 \mathrm{H}\right), 5.55\left(\mathrm{dd}, J^{\prime}=J^{\prime \prime}=7.8 \mathrm{~Hz}, 1 \mathrm{H}\right), 6.92(\mathrm{~d}, J=8.7$ $\mathrm{Hz}, 2 \mathrm{H}), 7.32$ (d, $J=8.7 \mathrm{~Hz}, 2 \mathrm{H}), 7.36$ (t, $J=7.2 \mathrm{~Hz}, 1 \mathrm{H}), 7.45$ (t, $J=7.2 \mathrm{~Hz}, 2 \mathrm{H}), 7.6-7.7$ (m, 4H), $7.96(\mathrm{~d}, J=8.1 \mathrm{~Hz}, 2 \mathrm{H})$. Anal. Calcd for $\mathrm{C}_{21} \mathrm{H}_{19} \mathrm{O}$ B B: C, 76.39; H, 5.80. Found: C, 76.27; H, 5.92.

(R)-(-)-2-(4-Biphenyl)-4-(4'-trifluoromethylphenyl)-1,3,2-dioxaborolane (2c): Yield 95\%; mp 91-93 ${ }^{\circ} \mathrm{C} ;[\alpha]_{\mathrm{D}}=-113.4(c=1.07, \mathrm{THF}){ }^{1} \mathrm{H}$ NMR $\left(300 \mathrm{MHz}, \mathrm{CDCl}_{3}\right) \delta 4.18\left(\mathrm{dd}, J^{\prime}=7.5 \mathrm{~Hz}, J^{\prime \prime}=\right.$ $8.9 \mathrm{~Hz}, 1 \mathrm{H}), 4.80\left(\mathrm{dd}, J^{\prime}=J^{\prime \prime}=8.7 \mathrm{~Hz}, 1 \mathrm{H}\right), 5.67\left(\mathrm{dd}, J^{\prime}=J^{\prime \prime}=7.9 \mathrm{~Hz}, 1 \mathrm{H}\right), 7.3-7.6(\mathrm{~m}, 5 \mathrm{H}), 7.6-$ $7.8(\mathrm{~m}, 6 \mathrm{H}), 7.97(\mathrm{~d}, J=8.2 \mathrm{~Hz}, 2 \mathrm{H})$. Anal. Calcd for $\mathrm{C}_{21} \mathrm{H}_{16} \mathrm{O}_{2} \mathrm{~B}: \mathrm{C}, 52.43 ; \mathrm{H}, 4.40$. Found: C, $52.37 ; \mathrm{H}, 4.66$.

(R)-(-)-2-(4-Biphenyl)-4-(4'-biphenyl)-1,3,2-dioxaborolane (2d): Yield 72\%; mp 159-162 C; $[\alpha]_{\mathrm{D}}=-141(c=1.0, \mathrm{THF}) ;{ }^{1} \mathrm{H}$ NMR $\left(300 \mathrm{MHz}\right.$, acetone- $\left.d_{6}\right) \delta 4.24\left(\mathrm{dd}, J^{\prime}=7.8 \mathrm{~Hz}, J^{\prime \prime}=8.8 \mathrm{~Hz}\right.$, $1 \mathrm{H}), 4.87\left(\mathrm{dd}, J^{\prime}=J^{\prime \prime}=8.6 \mathrm{~Hz}, 1 \mathrm{H}\right), 5.78\left(\mathrm{dd}, J^{\prime}=J^{\prime \prime}=7.8 \mathrm{~Hz}, 1 \mathrm{H}\right), 7.3-7.8(\mathrm{~m}, 16 \mathrm{H}), 7.97(\mathrm{~d}, J=$ 8.1 Hz, 2H). Anal. Calcd for $\mathrm{C}_{26} \mathrm{H}_{21} \mathrm{O}_{2} \mathrm{~B}: \mathrm{C}, 83.00$; H, 5.63. Found: C, 82.91; H, 5.75.

(R)-(-)-2-(4-Biphenyl)-4-(3'-bromophenyl)-1,3,2-dioxaborolane (2e): Yield 71\%; mp 52-53 ${ }^{\circ}$; $[\alpha]_{\mathrm{D}}=-120.5(c=0.57, \mathrm{THF}) ;{ }^{1} \mathrm{H}$ NMR $\left(300 \mathrm{MHz}, \mathrm{CDCl}_{3}\right) \delta 4.17\left(\mathrm{dd}, J^{\prime}=7.6 \mathrm{~Hz}, J^{\prime \prime}=9.0 \mathrm{~Hz}\right.$, 
1H), $4.75\left(\mathrm{dd}, J^{\prime}=J^{\prime \prime}=9.0 \mathrm{~Hz}, 1 \mathrm{H}\right), 5.57\left(\mathrm{dd}, J^{\prime}=J^{\prime \prime}=7.9 \mathrm{~Hz}, 1 \mathrm{H}\right), 7.2-7.7(\mathrm{~m}, 11 \mathrm{H}), 7.97(\mathrm{~d}, J=$ $8.2 \mathrm{~Hz}, 2 \mathrm{H}) .{ }^{13} \mathrm{C} \mathrm{NMR}\left(75 \mathrm{MHz}, \mathrm{CDCl}_{3}\right) \delta 73.2,78.2,122.9,124.1,126.7,127.2,127.7,128.7$, 128.8, 130.4, 131.3, 135.5, 140.9, 143.6, 144.5. Anal. Calcd for $\mathrm{C}_{20} \mathrm{H}_{16} \mathrm{O}_{2} \mathrm{BBr}: \mathrm{C}, 63.37$; H, 4.25. Found: C, 63.42; H, 4.36 .

(R)-(-)-2-(4-Biphenyl)-4-(2'-bromophenyl)-1,3,2-dioxaborolane (2f): Yield 80\%; mp 141$143^{\circ} \mathrm{C} ;[\alpha]_{\mathrm{D}}=-166.0(c=1.1, \mathrm{THF}) ;{ }^{1} \mathrm{H} \mathrm{NMR}\left(600 \mathrm{MHz}, \mathrm{CDCl}_{3}\right) \delta 4.15\left(\mathrm{dd}, J^{\prime}=7.1 \mathrm{~Hz}, J^{\prime \prime}=9.1\right.$ $\mathrm{Hz}, 1 \mathrm{H}), 4.98\left(\mathrm{dd}, J^{\prime}=8.4 \mathrm{~Hz}, J^{\prime}=9.1 \mathrm{~Hz}, 1 \mathrm{H}\right), 5.91\left(\mathrm{dd}, J^{\prime}=0.65 \mathrm{~Hz}, J^{\prime \prime}=7.0 \mathrm{~Hz}, J^{\prime \prime}{ }^{\prime}=8.4 \mathrm{~Hz}\right.$, 1H), $7.22\left(\mathrm{~m}, J^{\prime}=7.8 \mathrm{~Hz}, J^{\prime \prime}=1.8 \mathrm{~Hz}, 1 \mathrm{H}\right), 7.38\left(\right.$ br t $\left., J^{\prime}=7.8 \mathrm{~Hz}, J^{\prime \prime}=1.3 \mathrm{~Hz}, 1 \mathrm{H}\right), 7.40(\mathrm{~m}, J=$ $7.3 \mathrm{~Hz}, \mathrm{~J}=1.1 \mathrm{~Hz}, 1 \mathrm{H}), 7.49(\mathrm{~m}, \mathrm{~J} \approx 7.8 \mathrm{~Hz}, 2 \mathrm{H}), 7.595$ (br dd, $J^{\prime}=7.8 \mathrm{~Hz}, J^{\prime \prime}=1.8 \mathrm{~Hz}, J^{\prime \prime}{ }^{\prime}=0.65$ $\mathrm{Hz}, 1 \mathrm{H}), 7.605\left(\mathrm{dd}, J^{\prime}=7.8 \mathrm{~Hz}, J^{\prime \prime}=1.3 \mathrm{~Hz}, 1 \mathrm{H}\right), 7.68\left(\mathrm{~m}, J^{\prime}=7.9 \mathrm{~Hz}, J^{\prime \prime}=1.5 \mathrm{~Hz}, 2 \mathrm{H}\right), 7.70(\mathrm{~m}$, $J=8.2 \mathrm{~Hz}, 2 \mathrm{H}), 8.02(\mathrm{~m}, J=8.2 \mathrm{~Hz}, 2 \mathrm{H}) .{ }^{13} \mathrm{C} \mathrm{NMR}\left(75 \mathrm{MHz}, \mathrm{CDCl}_{3}\right) \delta 72.8,78.1,120.8,126.4$, $126.7,127.2,127.7,127.8,128.8,129.3,132.7,135.5,140.9,141.2$, 144.5. Anal. Calcd for $\mathrm{C}_{20} \mathrm{H}_{16} \mathrm{O}_{2} \mathrm{BBr}$ : C, 63.37; H, 4.25. Found: C, 63.40; H, 4.31.

(R)-(-)-2-(4-Biphenyl)-4-(2-naphthyl)-1,3,2-dioxaborolane (2g): Yield 80\%; mp 158-160 C; $[\alpha]_{\mathrm{D}}=-133.0(c=1.03, \mathrm{THF}) ;{ }^{1} \mathrm{H}$ NMR $\left(300 \mathrm{MHz}\right.$, acetone- $\left.d_{6}\right) \delta 4.28\left(\mathrm{dd}, J^{\prime}=7.6 \mathrm{~Hz}, J^{\prime \prime}=8.8\right.$ $\mathrm{Hz}, 1 \mathrm{H}), 4.90\left(\mathrm{dd}, J^{\prime}=J^{\prime \prime}=8.8 \mathrm{~Hz}, 1 \mathrm{H}\right), 5.90\left(\mathrm{dd}, J^{\prime}=J^{\prime \prime}=7.8 \mathrm{~Hz}, 1 \mathrm{H}\right), 7.3-7.8(\mathrm{~m}, 10 \mathrm{H}), 7.9-8.1$ (m, 6H). Anal. Calcd for $\mathrm{C}_{24} \mathrm{H}_{19} \mathrm{O}_{2} \mathrm{~B}: \mathrm{C}, 82.31 ; \mathrm{H}, 5.47$. Found: C, 82.27; H, 5.52.

(R)-(-)-2-(4-Biphenyl)-4-(1-naphthyl)-1,3,2-dioxaborolane (2h): Yield 72\%; $[\alpha]_{\mathrm{D}}=-232.5(c=$ 0.66, THF); ${ }^{1} \mathrm{H}$ NMR $\left(600 \mathrm{MHz}, \mathrm{CDCl}_{3}\right) \delta 4.22\left(\mathrm{dd}, J^{\prime}=7.4 \mathrm{~Hz}, J^{\prime \prime}=8.8 \mathrm{~Hz}, 1 \mathrm{H}\right), 5.04\left(\mathrm{dd}, J^{\prime}=\right.$ $\left.J^{\prime \prime}=8.6 \mathrm{~Hz}, 1 \mathrm{H}\right), 6.34\left(\mathrm{dd}, J^{\prime}=J^{\prime \prime}=7.9 \mathrm{~Hz}, 1 \mathrm{H}\right), 7.38(\mathrm{~m}, J=7.4 \mathrm{~Hz}, 1 \mathrm{H}), 7.47(\mathrm{~m}, J=7.4 \mathrm{~Hz}$, 2H), $7.50(\mathrm{dd}, J=7.9 \mathrm{~Hz}, 1 \mathrm{H}), 7.54(\mathrm{~m}, 2 \mathrm{H}), 7.66(\mathrm{~m}, J=7.4 \mathrm{~Hz}, 2 \mathrm{H}), 7.69(\mathrm{~m}, J=8.2 \mathrm{~Hz}, 2 \mathrm{H})$, $7.74\left(\mathrm{~m}, J^{\prime}=7.9 \mathrm{~Hz}, J^{\prime \prime}=1,2 \mathrm{~Hz}, 1 \mathrm{H}\right), 7.77$ (br d, $\left.J=8.1 \mathrm{~Hz}, 1 \mathrm{H}\right), 7.83$ (d, J=8.0 Hz, 1H), 7.92 (br $\left.\mathrm{dd}, J^{\prime}=7.5 \mathrm{~Hz}, 1 \mathrm{H}\right), 8.05(\mathrm{~m}, J=8.2 \mathrm{~Hz}, 2 \mathrm{H}) .{ }^{13} \mathrm{C} \mathrm{NMR}\left(75 \mathrm{MHz}, \mathrm{CDCl}_{3}\right) \delta 73.1,121.9,122.5$, $125.6,125.8,126.4,126.7,127.3,127.7,128.3,128.8,129.1,129.9$, 133.8, 135.6, 137.2, 140.9, 144.4. Anal. Calcd for $\mathrm{C}_{24} \mathrm{H}_{19} \mathrm{O}_{2} \mathrm{~B}: \mathrm{C}, 82.31 ; \mathrm{H}$, 5.47. Found: $\mathrm{C}, 82.34 ; \mathrm{H}, 5.50$.

(R)-(+)-2-(4-Biphenyl)-4-(9-anthryl)-1,3,2-dioxaborolane (2i): Yield 57\%; $[\alpha]_{\mathrm{D}}=+29.9(c=1.0$, THF); ${ }^{1} \mathrm{H}$ NMR $\left(300 \mathrm{MHz}, \mathrm{CDCl}_{3}\right) \delta 4.75\left(\mathrm{dd}, J^{\prime}=J^{\prime \prime}=9.2 \mathrm{~Hz}, 1 \mathrm{H}\right), 4.95\left(\mathrm{dd}, J^{\prime}=J^{\prime \prime}=9.2 \mathrm{~Hz}\right.$, $1 \mathrm{H}), 7.13\left(\mathrm{dd}, J^{\prime}=J^{\prime \prime}=9.4 \mathrm{~Hz}, 1 \mathrm{H}\right), 7.3-7.5(\mathrm{~m}, 6 \mathrm{H}), 7.66(\mathrm{~d}, J=8.8 \mathrm{~Hz}, 2 \mathrm{H}), 7.68(\mathrm{~d}, J=8.5 \mathrm{~Hz}$, 2H), $7.78(\mathrm{~m}, 1 \mathrm{H}), 8.05(\mathrm{~d}, J=7.1 \mathrm{~Hz}, 4 \mathrm{H}), 8.38(\mathrm{~d}, J=8.1 \mathrm{~Hz}, 2 \mathrm{H}), 8.51(\mathrm{~s}, 1 \mathrm{H})$. Anal. Calcd for $\mathrm{C}_{28} \mathrm{H}_{21} \mathrm{O}_{2} \mathrm{~B}: \mathrm{C}, 84.02$; H, 5.29. Found: C, 84.10; H, 5.23. 
(4R,5S)-(-)-2-(4-Biphenyl)-4-phenyl-5-carboxymethyl-1,3,2-dioxaborolane (2j): Yield 90\%; $[\alpha]_{\mathrm{D}}=-77.2(c=1.0, \mathrm{THF}) ;{ }^{1} \mathrm{H} \mathrm{NMR}\left(300 \mathrm{MHz} \mathrm{CDCl}_{3}\right) \delta 3.9(\mathrm{~s}, 3 \mathrm{H}), 4.87(\mathrm{~d}, J=5.7 \mathrm{~Hz}, 1 \mathrm{H})$, $5.62(\mathrm{~d}, J=5.7 \mathrm{~Hz}, 1 \mathrm{H}), 7.3-7.5(\mathrm{~m}, 8 \mathrm{H}), 7.65(\mathrm{~m}, 4 \mathrm{H}), 8.02(\mathrm{~d}, J=7.4 \mathrm{~Hz}, 2 \mathrm{H}) ;{ }^{13} \mathrm{C}$ NMR $(75$ $\left.\mathrm{MHz}, \mathrm{CDCl}_{3}\right) \delta 52.7,81.9,82.6,125.3,126.7,126.8,127.3,127.7,128.5,128.9,135.8,136.2$, 140.4, 140.8, 144.7, 171.0. Anal. Calcd for $\mathrm{C}_{22} \mathrm{H}_{19} \mathrm{O}_{4} \mathrm{~B}$ : C, 73.77; H, 5.35. Found: C, 73.78; H, 5.31.

(4R,5R)-(-)-2-(4-Biphenyl)-4-(4'-methoxyphenyl)-5-methyl-1,3,2-dioxaborolane (2k): Yield $77 \%$; glassy solid; $[\alpha]_{\mathrm{D}}=-105.8(c=1.05, \mathrm{THF}) ;{ }^{1} \mathrm{H} \mathrm{NMR}\left(300 \mathrm{MHz}, \mathrm{CDCl}_{3}\right) \delta 1.51(\mathrm{~d}, J=6.2$ $\mathrm{Hz}, 3 \mathrm{H}), 3.81(\mathrm{~s}, 3 \mathrm{H}), 4.47\left(\mathrm{dq}, J^{\prime}=J^{\prime \prime}=6.2 \mathrm{~Hz}, 1 \mathrm{H}\right), 5.00(\mathrm{~d}, J=7.6 \mathrm{~Hz}, 1 \mathrm{H}), 6.93(\mathrm{~d}, J=8.6 \mathrm{~Hz}$, 1H), $7.33(\mathrm{~d}, J=8.6 \mathrm{~Hz}, 2 \mathrm{H}), 7.36(\mathrm{~m}, 1 \mathrm{H}), 7.46(\mathrm{t}, J=7.8 \mathrm{~Hz}, 2 \mathrm{H}), 7.65(\mathrm{~d}, J=8.6 \mathrm{~Hz}, 4 \mathrm{H}), 7.96$ $(\mathrm{d}, J=7.9 \mathrm{~Hz}, 2 \mathrm{H})$. Anal. Calcd for $\mathrm{C}_{22} \mathrm{H}_{21} \mathrm{O}_{3} \mathrm{~B}: \mathrm{C}, 76.77$; H, 6.15. Found: C, 76.58; H, 6.23.

(4R,5S)-(-)-2-(4-Biphenyl)-4-(4'-methoxyphenyl)-5-methyl-1,3,2-dioxaborolane (2l): Yield $73 \%$; glassy solid; ${ }^{1} \mathrm{H}$ NMR $\left(300 \mathrm{MHz}, \mathrm{CDCl}_{3}\right) \delta 0.94(\mathrm{~d}, J=6.5 \mathrm{~Hz}, 3 \mathrm{H}), 3.81(\mathrm{~s}, 3 \mathrm{H}), 4.96$ (dq, $J$ ' $\left.=6.5 \mathrm{~Hz}, J^{\prime \prime}=7.7 \mathrm{~Hz}, 1 \mathrm{H}\right), 5.63(\mathrm{~d}, J=7.7 \mathrm{~Hz}, 1 \mathrm{H}), 6.90(\mathrm{~d}, J=8.6 \mathrm{~Hz}, 2 \mathrm{H}), 7.20(\mathrm{~d}, J=8.6 \mathrm{~Hz}$, 2H), 7.3-7.6 (m, 3H), $7.64(\mathrm{~d}, J=7.1 \mathrm{~Hz}, 2 \mathrm{H}), 7.66(\mathrm{~d}, J=8.0 \mathrm{~Hz}, 2 \mathrm{H}), 7.98(\mathrm{~d}, J=8.0 \mathrm{~Hz}, 2 \mathrm{H})$. Anal. Calcd for $\mathrm{C}_{22} \mathrm{H}_{21} \mathrm{O}_{3}$ B: C, 76.77; H, 6.15. Found: C, 76.68; H, 6.18.

(4R,5R)-(-)-2-(4-Biphenyl)-4-(2-thienyl)-5-carboxymethyl-1,3,2-dioxaborolane (2m): Yield $80 \% ;[\alpha]_{\mathrm{D}}=+60.0(c=1.0, \mathrm{THF}) ;{ }^{1} \mathrm{H} \mathrm{NMR}\left(300 \mathrm{MHz}, 300 \mathrm{MHz}, \mathrm{CDCl}_{3}\right) \delta 3.86(\mathrm{~s}, 3 \mathrm{H}), 5.00(\mathrm{~d}, J$ $=6.3 \mathrm{~Hz}, 1 \mathrm{H}), 5.84(\mathrm{~d}, J=6.4 \mathrm{~Hz}, 1 \mathrm{H}), 7.03(\mathrm{dd}, \mathrm{J}$ ' = $3.4 \mathrm{~Hz}, \mathrm{~J}$ ' = 5.0 Hz, 1H), 7.3-7.5 (m, 4H), $7.66(\mathrm{~d}, \mathrm{~J}=6.3,2 \mathrm{H}), 7.63(\mathrm{~d}, \mathrm{~J}=6.6,2 \mathrm{H}), 8.00(\mathrm{~d}, J=8.1 \mathrm{~Hz}, 2 \mathrm{H}) ;{ }^{13} \mathrm{C} \mathrm{NMR}\left(75 \mathrm{MHz}, \mathrm{CDCl}_{3}\right) \delta$ 52.7, 78.4, 82.5, 125.0, 125.9, 126.7, 127.1, 127.3, 127.7, 128.8, 135.8, 140.8, 143.4, 144.8, 170.1 . Anal. Calcd for $\mathrm{C}_{20} \mathrm{H}_{17} \mathrm{O}_{4} \mathrm{BS}$ : C, 65.95; H, 4.70. Found: C, 65.88; H, 4.68.

(4S,5R)-(-)-2-(4-Biphenyl)-8,8a-dihydro-3a $H$-indeno[1,2-d][1,3,2]dioxaborole (2n): Yield 77\%; mp 81-83 ${ }^{\circ} \mathrm{C} ;[\alpha]_{\mathrm{D}}=-29.9\left(c=0.97, \mathrm{CHCl}_{3}\right) ;{ }^{1} \mathrm{H} \mathrm{NMR}\left(300 \mathrm{MHz}, \mathrm{CDCl}_{3}\right) \delta 3.30(\mathrm{~d}, J=17.5 \mathrm{~Hz}$, $1 \mathrm{H}) ; 3.43\left(\mathrm{dd}, J^{\prime}=6.6 \mathrm{~Hz}, J^{\prime \prime}=17.5,1 \mathrm{H}\right), 5.38\left(\mathrm{dt}, J^{\prime}=6.6 \mathrm{~Hz}, J^{\prime \prime}=1.5 \mathrm{~Hz}, 1 \mathrm{H}\right), 5.91(\mathrm{~d}, J=6.6$ $\mathrm{Hz}, 1 \mathrm{H})), 7.2-7.5(\mathrm{~m}, 6 \mathrm{H}), 7.5-7.7(\mathrm{~m}, 5 \mathrm{H}), 7.86(\mathrm{~d}, J=7.9 \mathrm{~Hz}, 1 \mathrm{H}) ;{ }^{13} \mathrm{C} \mathrm{NMR}\left(75 \mathrm{MHz}, \mathrm{CDCl}_{3}\right) \delta$ 39.9, 81.0, 85.1, 125.4, 126.2, 126.5, 127.2, 127.5, 127.6, 128.8, 129.6, 135.3, 140.4, 140.8, 140.9, 144.0. Anal. Calcd for $\mathrm{C}_{21} \mathrm{H}_{17} \mathrm{O}_{2} \mathrm{~B}: \mathrm{C}, 80.80 ; \mathrm{H}, 5.49$. Found: C, 80.76; H, 5.43 .

\section{(4S,5R)-(-)-2-(4-Biphenyl)-3a,4,5,9b-tetrahydro-1,3-dioxa-2-bora-cyclopenta[a]naphthalene}

(2o): Yield 77\%; mp $107-108^{\circ} \mathrm{C} ;[\alpha]_{\mathrm{D}}=-23.8(c=1.0, \mathrm{THF}) ;{ }^{1} \mathrm{H}$ NMR $\left(300 \mathrm{MHz}, \mathrm{CDCl}_{3}\right) \delta 1.9-$ $2.1(\mathrm{~m}, 1 \mathrm{H}), 2.0-2.3(\mathrm{~m}, 1 \mathrm{H}), 2.5-2.7(\mathrm{~m}, 1 \mathrm{H}), 2.8-3.0(\mathrm{~m}, 1 \mathrm{H}), 5.06\left(\mathrm{dt}, J^{\prime}=4.7 \mathrm{~Hz}, J^{\prime \prime}=7.8 \mathrm{~Hz}\right.$, 
1H), $5.56(\mathrm{~d}, J=7.8 \mathrm{~Hz}, 1 \mathrm{H}) ; 7.1-7.7(\mathrm{~m}, 10 \mathrm{H}), 7.89(\mathrm{~d}, J=8.1 \mathrm{~Hz}, 2 \mathrm{H}) ;{ }^{13} \mathrm{C} \mathrm{NMR}(75 \mathrm{MHz}$, $\left.\mathrm{CDCl}_{3}\right) \delta 42.9,43.9,74.8,117.9,126.6,127.1,127.5,128.0,128.2,128.3,129.5,129.6,130.2$, 135.9, 136.1, 138.29, 140.2, 140.3. Anal. Calcd for $\mathrm{C}_{22} \mathrm{H}_{19} \mathrm{O}_{2} \mathrm{~B}: \mathrm{C}, 81.01$; H, 5.87. Found: C, 81.07; $\mathrm{H}, 5.83$.

Table Ratios of computed interproton distances and ratios between experimental NOEs detected in the aromatic region for $\mathbf{2 f}$.

\begin{tabular}{cccc}
\hline $\mathrm{NOE}^{a, b}$ & Ratio & Compd Dist $(\AA)$ & Ratio \\
\hline${\text { (HaH6/HaHo })^{1 / 6}}^{1 / 6}$ & 1.22 & $\mathrm{HaHo} / \mathrm{HaH6}$ & 1.29 \\
$(\mathrm{HaH6} / \mathrm{HaH} 3)^{1 / 6}$ & 1.30 & $\mathrm{HaH} 3 / \mathrm{HaH} 6$ & 1.33 \\
$(\mathrm{HaH} 3 / \mathrm{HaHo})^{1 / 6}$ & 0.94 & $\mathrm{HaHo} / \mathrm{HaH} 3$ & 0.97 \\
$(\mathrm{HaHo} / \mathrm{HbHo})^{1 / 6}$ & 1.06 & $\mathrm{HbHo} / \mathrm{HaHo}$ & 1.10 \\
$(\mathrm{HcHo} / \mathrm{HaHo})^{1 / 6}$ & 0.98 & $\mathrm{HaHo} / \mathrm{HcHo}$ & 0.99 \\
$(\mathrm{HcH} / \mathrm{HcHo})^{1 / 6}$ & 1.20 & $\mathrm{HcHo} / \mathrm{HcH6}$ & 1.53 \\
$(\mathrm{HcH} 3 / \mathrm{HcHo})^{1 / 6}$ & 0.88 & $\mathrm{HcHo} / \mathrm{HcH} 3$ & 0.79 \\
${ }^{a}$ In the reported ratios between couples of protons the first refers to the \\
irradiated one whereas the second refers to the observed proton. ${ }^{b} \mathrm{Ho}$ refers \\
to the biphenyl ortho protons at 8.02 ppm
\end{tabular}

PCModel 7.0 (Serena Software, Bloomington, IN 47402-3076, U.S.A)

Molecular Mechanics (MMX) Full Optimised Geometries.

$\underline{\text { Cartesian coordinates for compound }(R)-2 \mathbf{i}(\mathrm{MMX} \text { Energy }=56.798 \mathrm{kcal} / \mathrm{mol})}$
C $\quad-2.317145 \quad 0.610145 \quad 2.711686$
C $\quad-1.961537 \quad 2.054734 \quad 3.088222$
$\begin{array}{llll}\mathrm{O} & -0.540019 & 2.075105 & 3.085861\end{array}$
B $\quad-0.188370 \quad 1.063019 \quad 2.310549$
O $-1.168100 \quad 0.249755 \quad 1.950571$
C $\quad 1.607809-0.243211 \quad 1.015871$
C $\quad 1.283774 \quad 0.837345 \quad 1.847271$
C $\quad 2.299347 \quad 1.711973 \quad 2.255750$
C $3.620093 \quad 1.507393 \quad 1.841949$ 

C $\quad 3.950507 \quad 0.426816 \quad 1.006917$
$\begin{array}{llll}\text { C } & 2.927575 & -0.445314 & 0.597908\end{array}$
$\begin{array}{llll}\text { C } & 7.470883 & 1.089287 & -0.274213\end{array}$
$\begin{array}{llll}\text { C } & 6.149726 & 1.291707 & 0.139415\end{array}$
$\begin{array}{llll}\text { C } & 5.358672 & 0.209954 & 0.563231\end{array}$
C $5.917770 \quad-1.079666 \quad 0.561654$
C $7.237216-1.283832 \quad 0.143415$
$\begin{array}{llll}\text { C } & 8.017139 & -0.199441 & -0.274609\end{array}$
$\begin{array}{llll}\mathrm{H} & -2.325216 & 2.396147 & 4.087361\end{array}$
H $\quad-2.325238 \quad 2.766197 \quad 2.303895$
H $\quad 0.822488 \quad-0.9409690 .680807$
$\mathrm{H} \quad 2.064925 \quad 2.567176 \quad 2.910845$
$\mathrm{H} \quad 4.401768 \quad 2.201987 \quad 2.191596$
H $3.151265-1.294552-0.068885$
H $\quad 8.082636 \quad 1.945879 \quad-0.604068$
H $\quad 5.734457 \quad 2.312937 \quad 0.116660$
H $\quad 5.326202 \quad-1.945850 \quad 0.901661$
H $7.665113-2.300546 \quad 0.145788$
H $\quad 9.058289 \quad-0.359635 \quad-0.602335$
H $\quad-2.343729 \quad-0.029964 \quad 3.630454$
C $\quad-4.931031 \quad 1.693683 \quad 3.723756$
$\begin{array}{llll}\text { C } & -5.980700 & 2.512839 & 4.056862\end{array}$
C $\quad-6.976286 \quad 2.852529 \quad 3.085886$
$\begin{array}{llll}\text { C } & -6.918880 & 2.313662 & 1.825991\end{array}$
$\begin{array}{llll}\text { C } & -5.839969 & 1.431178 & 1.460317\end{array}$
$\begin{array}{llll}\text { C } & -4.778037 & 1.182741 & 2.383330\end{array}$
$\begin{array}{llll}\text { C } & -5.817998 & 0.824466 & 0.193477\end{array}$
C $\quad-3.634716 \quad 0.456903 \quad 1.965070$
$\begin{array}{llll}\text { C } & -3.753388 & -0.355879 & 0.806698\end{array}$
C $-4.811642-0.105973-0.121636$
C $\quad-2.858502 \quad-1.446530 \quad 0.503403$
C $-4.840855-0.796221-1.385801$
C $\quad-2.919602-2.107869-0.698257$
C $\quad-3.906952-1.758898-1.672604$
H $\quad-4.225414 \quad 1.410691 \quad 4.520190$ 

H $\quad-6.067789 \quad 2.908207 \quad 5.083108$
H $\quad-7.800186 \quad 3.532423 \quad 3.360936$
$\mathrm{H} \quad-7.709411 \quad 2.559753 \quad 1.097043$
H $\quad-6.612931 \quad 1.046907 \quad-0.537785$
$\mathrm{H} \quad-2.121120 \quad-1.802947 \quad 1.238921$
H $\quad-5.617126 \quad-0.559600 \quad-2.133009$
H $-2.208582-2.923175-0.915609$
H $\quad-3.927548 \quad-2.280708 \quad-2.644324$

$\underline{\text { Cartesian coordinates for compound }(4 S, 5 R)-2 \mathrm{n}(\mathrm{MMX} \text { Energy }=37.593 \mathrm{kcal} / \mathrm{mol})}$
C $\quad-5.528550-1.167550 \quad 1.396300$
C $\quad-6.197350-0.469550 \quad 2.413100$
C $\quad-6.621850 \quad 0.853150 \quad 2.216200$
C $\quad-6.390750 \quad 1.507250 \quad 0.996600$
C $\quad-5.727850 \quad 0.802050 \quad-0.009100$
C $\quad-5.299250-0.504750 \quad 0.189300$
C $\quad-5.392350 \quad 1.310250 \quad-1.377500$
C $-4.602850-1.064550-1.016800$
C $\quad-4.434750 \quad 0.199850 \quad-1.883000$
O $\quad-3.280550 \quad-1.496150 \quad-0.751400$
B $-2.499650-0.477350-1.074400$
$\begin{array}{llll}\text { O } & -3.080550 & 0.565450 & -1.648200\end{array}$
C $\quad 1.003450-1.644650 \quad 0.064100$
C $\quad-0.372250-1.620250-0.186900$
C $\quad-0.965050-0.503450-0.792000$
C $\quad-0.159650 \quad 0.588750 \quad-1.138900$
C $\quad 1.216250 \quad 0.566050 \quad-0.883600$
C $\quad \begin{array}{llll}1.817350 & -0.551150 & -0.279700\end{array}$
$\begin{array}{llll}\text { C } & 3.285950 & -0.576350 & -0.009300\end{array}$
$\begin{array}{llll}\text { C } & 4.196250 & -0.043350 & -0.938400\end{array}$
$\begin{array}{llll}\text { C } & 5.572450 & -0.064650 & -0.684400\end{array}$
C $\quad 3.787450-1.134150 \quad 1.180600$
C $\quad 5.162650-1.160350 \quad 1.433700$ 

C $\quad 6.059350 \quad-0.624350 \quad 0.501700$
$\mathrm{H} \quad-5.189150 \quad-2.206050 \quad 1.543100$
H $\quad-6.389850 \quad-0.967150 \quad 3.378600$
$\mathrm{H} \quad-7.144050 \quad 1.385650 \quad 3.029000$
$\mathrm{H} \quad-6.722950 \quad 2.545550 \quad 0.832900$
H $\quad-4.920950 \quad 2.320250 \quad-1.360000$
$\mathrm{H} \quad-6.325050 \quad 1.337650 \quad-1.986600$
$\mathrm{H} \quad 1.440550 \quad-2.545550 \quad 0.525300$
H $\quad-0.987250 \quad-2.491450 \quad 0.093100$
$\mathrm{H} \quad-0.605450 \quad 1.478550 \quad-1.613300$
H $\quad 1.818450 \quad 1.449250 \quad-1.153200$
H $\quad 3.840450 \quad 0.387650 \quad-1.888700$
$\mathrm{H} \quad 6.275650 \quad 0.357250 \quad-1.422200$
H $\quad 3.102150 \quad-1.547150 \quad 1.939100$
H $\quad 5.542450 \quad-1.600950 \quad 2.371200$
H $\quad 7.144050 \quad-0.643050 \quad 0.701700$
Н $\quad-5.426650 \quad-2.181050 \quad-1.661300$
$\mathrm{H} \quad-4.624050 \quad-0.075450 \quad-3.378600$

$\underline{\text { Cartesian coordinates for compound }(4 S, 5 R)-\mathbf{2 0}(\mathrm{MMX} \text { Energy }=35.399 \mathrm{kca} / \mathrm{mol})}$
C $\quad-5.279150 \quad-1.498550 \quad 1.357100$
C $\quad-6.243450 \quad-1.174150 \quad 2.318200$
$\begin{array}{llll}\text { C } & -7.045050 & -0.043150 & 2.141800\end{array}$
C $\quad-6.874250 \quad 0.760250 \quad 1.008100$
C $\quad-5.905850 \quad 0.442550 \quad 0.045200$
C $\quad-5.106250-0.700450 \quad 0.217500$
C $\quad-5.727250 \quad 1.334550-1.156200$
C $\quad-4.066850 \quad-1.095150 \quad-0.802100$
C $\quad-3.953950 \quad-0.201750 \quad-2.033300$
$\begin{array}{llll}\text { O } & -2.748650 & -1.065650 & -0.269600\end{array}$
B $\quad-1.968250 \quad-0.716050-1.283500$
O $\quad-2.582350-0.318750-2.389800$
C $\quad 1.602650-1.225750 \quad 0.100300$ 

C $\quad 0.207550 \quad-1.180250 \quad 0.007000$
C $-0.412350-0.764750-1.179000$
$\begin{array}{llll}\text { C } & 0.385050 & -0.392650 & -2.269200\end{array}$
C $\quad 1.780550 \quad-0.433750 \quad-2.174600$
C $\quad 2.408550 \quad-0.851950 \quad-0.989000$
C $3.896450 \quad-0.898150 \quad-0.888900$
C $4.673850-1.360350 \quad-1.965300$
C $6.069550-1.401750 \quad-1.872800$
$\begin{array}{llll}\text { C } & 4.549750 & -0.480150 & 0.284200\end{array}$
C $\quad 5.944850 \quad-0.525150 \quad 0.379600$
$\begin{array}{llll}\text { C } & 6.708650 & -0.985250 & -0.699400\end{array}$
C $\quad-4.296350 \quad 1.257050 \quad-1.696900$
H $\quad-4.650450 \quad-2.393250 \quad 1.501300$
$\mathrm{H} \quad-6.371450 \quad-1.809250 \quad 3.211100$
$\mathrm{H} \quad-7.808750 \quad 0.216050 \quad 2.894600$
$\mathrm{H} \quad-7.511650 \quad 1.650850 \quad 0.878900$
$\begin{array}{llll}\mathrm{H} & -5.960350 & 2.393250 & -0.892200\end{array}$
H $\quad-6.453150 \quad 1.014650 \quad-1.940300$
H $\quad-4.283950-2.141950-1.138900$
H $\quad-4.599950 \quad-0.556550 \quad-2.877300$
$\mathrm{H} \quad 2.059650 \quad-1.573250 \quad 1.041700$
H $\quad-0.401350 \quad-1.477950 \quad 0.876700$
H $\quad-0.082350 \quad-0.060950 \quad-3.211100$
H $\quad 2.380050 \quad-0.118850 \quad-3.044700$
H $\quad 4.193850 \quad-1.709650 \quad-2.894400$
H $\quad 6.667950 \quad-1.766550 \quad-2.724800$
H $\quad 3.971850 \quad-0.098150 \quad 1.141900$
H $\quad 6.445150 \quad-0.194550 \quad 1.305600$
H $7.808750-1.019350-0.625300$
$\mathrm{H} \quad-3.587650 \quad 1.656350 \quad-0.932000$
$\begin{array}{llll}\mathrm{H} & -4.193750 & 1.892450 & -2.609200\end{array}$ 